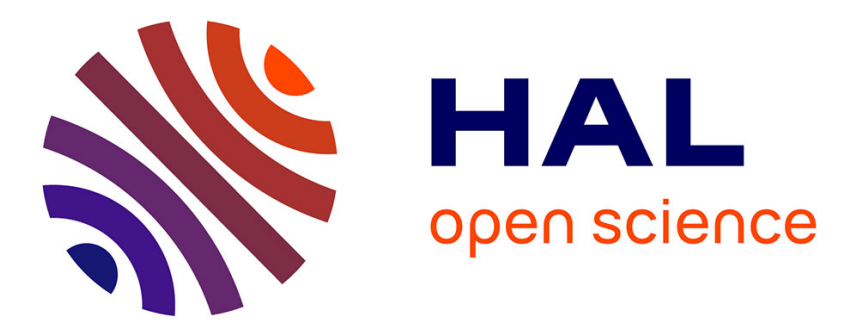

\title{
Representing Synergy among Arguments with Choquet Integral
}

Souhila Kaci, Christophe Labreuche

\section{To cite this version:}

Souhila Kaci, Christophe Labreuche. Representing Synergy among Arguments with Choquet Integral. ECSQARU: European Conference on Symbolic and Quantitative Approaches to Reasoning and Uncertainty, Jul 2013, Utrecht, Netherlands. pp.302-314, 10.1007/978-3-642-39091-3_26 . lirmm00857796

\section{HAL Id: lirmm-00857796 https://hal-lirmm.ccsd.cnrs.fr/lirmm-00857796}

Submitted on 14 Sep 2019

HAL is a multi-disciplinary open access archive for the deposit and dissemination of scientific research documents, whether they are published or not. The documents may come from teaching and research institutions in France or abroad, or from public or private research centers.
L'archive ouverte pluridisciplinaire HAL, est destinée au dépôt et à la diffusion de documents scientifiques de niveau recherche, publiés ou non, émanant des établissements d'enseignement et de recherche français ou étrangers, des laboratoires publics ou privés. 


\title{
Representing Synergy among Arguments with Choquet Integral
}

\author{
Souhila $\mathrm{Kaci}^{1}$ and Christophe Labreuche ${ }^{2}$ \\ ${ }^{1}$ LIRMM - UMR 5506, Montpellier, France \\ kaci@lirmm.fr \\ 2 Thales Research \& Technology, Palaiseau, France \\ christophe. labreuche@ thalesgroup.com
}

\begin{abstract}
Preference-based argumentation frameworks are instantiation of Dung's framework in which the defeat relation (in the sense of Dung) is computed from an attack relation and a preference relation over the set of arguments. Valuebased argumentation framework is a preference-based argumentation framework where the preference relation over arguments is derived from a preference relation over values they promote. We extend value-based argumentation framework with collective defeats and arguments promoting values with various strengths. In the extended framework, we define a function which computes the strength of a collective defeat. We define desired properties for the proposed function. Surprisingly, we show that this function obeying the corresponding properties is Choquet integral, a well-known aggregation function at work in multiple criteria decision.
\end{abstract}

\section{Introduction}

Argumentation is a reasoning framework which consists first in constructing the arguments, then identifying the acceptable ones and finally drawing conclusions. Dung has proposed an abstract argumentation framework that is composed of a set of arguments and a binary relation which is interpreted as a defeat relation between the arguments [8]. Two basic properties are used: conflict-freeness and defense. These two concepts define the output of an argumentation framework which is a set of sets of arguments that can be accepted together.

Dung's argumentation framework is said abstract as arguments and defeat relation are abstract, i.e. their origin is not known. This had the advantage to see this framework instantiated or extended in different ways. For example a noticeable extension consists of combined defeats: Several arguments may interact and entail a stronger defeat than each can do individually [21]. Dung's framework has also been instantiated with preferences. It is commonly acknowledged that preferences play an important role to solve conflicts between arguments. Preference-based argumentation frameworks are instantiation of Dung's framework in which the defeat relation is derived from an attack relation between arguments and a preference relation over the arguments [24, 1- 3, 14, 12]. An attack succeeds (thus called a defeat) if the attacked argument is not strictly preferred to the attacking one. Different ways have been proposed to compute a preference relation over the arguments. For example, the latter may promote different values which may be decisions, point of views, actions, etc. From the audience's preference relation over 
the values, one can derive a preference relation over the arguments. This framework is called value-based argumentation framework [3].

Why should several arguments interact? The basic idea of the paper is that interaction may actually arise from synergy among values supported by arguments. Synergies among values as felt by the audience are easier to elicit than directly interaction among arguments. Hence we extend value-based argumentation framework with collective defeats with varied strengths. Consider the following example.

Example 1 (Humanitarian action in Africa). In a small village, there is no well so that inhabitants have to go quite far away to get water. In order to help inhabitants, a humanitarian association decided to construct a well inside the village. Actually this action has turned the population against the association for the following reasons:

- There was a local economy around the transportation of water from the remote well. The construction of the well has turned this economy into bankruptcy. These people become hostile to the association.

- As water became an easily accessible resource, people started to waste it. Yet in an area that suffers from severe drought, water is a scarce resource and its waste endangers the equilibrium of the whole area.

- The decision from the association has been seen as interference because local authority has not been sufficiently consulted.

There are several values involved here: $\mathcal{V}=\{$ health, eco, env, pol $\}$, where health, eco, env and pol respectively stand for health, economy, environment and political stability. We assume that the values in the previous list are ordered from the most preferred one to the least preferred one. The following arguments can be defined:

- $a$ : Construct the well to help the village solve the water problem. It promotes value health.

- $b$ : Do not construct the well in order to avoid turning local economy into bankruptcy. It promotes value eco.

- $c$ : Do not construct the well in order to avoid water waste. It promotes value $e n v$.

- $d$ : Do not construct the well in order to avoid interference. The fact that the local authority has not been sufficiently consulted might weaken a little bit its stability. But this will by no mean deeply undermine political stability. Hence argument $d$ promotes only partly value pol.

Argument $a$ is in conflict with any argument $b, c$ and $d$. In this example, argument $a$ is stronger than any other argument $b, c, d$ as it promotes the most important value. Hence the single attacks of $b, c$ and $d$ on $a$ are not sufficient to undermine $a$, whereas $a$ defeats any of the three arguments $b, c, d$. However, arguments $b, c, d$ together promote three values that (considered together) may be stronger than value health. Hence the combined attack of $b, c$ and $d$ on $a$ may convince the audience. In this paper, we propose an argumentation framework which handles such considerations. The basic ingredient will be the concept of capacity to represent the potential interaction among values.

The rest of the paper is structured as follows. In the next two sections we recall Dung's argumentation framework and its main instantiations/extensions. Subsection 3.2 is however novel. It extends collective argumentation framework (in which the defeat 
relation is defined between sets of arguments) with a varied strength defeat relation. In Section 4 we extend value-based argumentation framework with collective and varied strength defeat relations. The new framework is based on a function to model interaction among values. Surprisingly, we show that this function obeying some properties is the Choquet integral, a well-known multiple criteria aggregation function. Lastly we conclude.

\section{Argumentation Theory}

\subsection{Dung's Argumentation Framework}

Argumentation is a reasoning model based on constructing arguments, determining potential conflicts between arguments and selecting acceptable arguments. In Dung's framework, arguments are supposed to be given. Conflicts between arguments are represented by a binary defeat relation.

Definition 1. [8] An argumentation framework $(A F)$ is a tuple $\langle\mathcal{A}, \rightarrow\rangle$ where $\mathcal{A}$ is a finite set of arguments and $\rightarrow \subseteq \mathcal{A} \times \mathcal{A}$ is a binary defeat relation.

The outcome of Dung's argumentation framework is sets of arguments, called extensions, that are robust against defeats. We say that $A \subseteq \mathcal{A}$ defends $a$ if $\forall b \in \mathcal{A}$ s.t. $b \rightarrow$ $a, \exists c \in A$ such that $c \rightarrow b$. We say that $A \subseteq \mathcal{A}$ is conflict-free if there are no $a, b \in A$ such that $a \rightarrow b$. A subset $A \subseteq \mathcal{A}$ of arguments is an admissible extension iff it is conflict-free and it defends all elements in $A$. Other acceptability semantics exist [8].

\subsection{Preference-Based Argumentation Framework}

Preference-based argumentation framework is an instantiation of Dung's framework. It is based on a binary attack relation between arguments and a preference relation over the set of arguments.

Definition 2. [1] A preference-based argumentation framework (PAF) is a 3-tuple $\langle\mathcal{A}, \rightsquigarrow, \succeq\rangle$ where $\mathcal{A}$ is a set of arguments, $\rightsquigarrow \subseteq \mathcal{A} \times \mathcal{A}$ is a binary attack relation and $\succeq$ is a preorder over $\mathcal{A}$.

$\succeq$ is called a Boolean preference relation. $\operatorname{A} \operatorname{PAF}\langle\mathcal{A}, \rightsquigarrow, \succeq\rangle$ represents $\langle\mathcal{A}, \rightarrow\rangle$ iff

$$
\forall a, b \in \mathcal{A}: \quad a \rightarrow b \text { iff }(a \rightsquigarrow b \text { and } \operatorname{not}(b \succ a))
$$

where $b \succ a$ is true if and only if $b \succeq a$ holds but $a \succeq b$ does not.

The extensions of a PAF are simply the extensions of the AF it represents.

Different ways have been proposed in the literature to compute the preference relation $\succeq$ over $\mathcal{A}$. For example, a weight function $w: \mathcal{A} \rightarrow[0,1]$ can be defined. Then

$$
\forall a, b \in \mathcal{A}: a \succeq b \text { iff } w(a) \geq w(b) .
$$

In some applications, the arguments need to be compared not on the basis of their internal structure but with respect to the viewpoints or decisions they promote [3]. This may be due to the fact that the internal structure of the arguments is not available or 
because the values must be considered. This is particularly true in persuasion dialogs when the preference over values induces the preference over arguments promoting the values [3]. Thus, if two arguments are conflicting then the argument promoting a preferred value is accepted. Bench-Capon developed an argumentation framework which models the above considerations [3]. Like Dung's framework, he considers abstract arguments. Moreover, he considers (i) a set of values promoted by the arguments and (ii) a set of audiences where an audience corresponds to a preference relation over values.

Definition 3. [3] A value-based argumentation framework is a five-tuple, VAF= $\langle\mathcal{A}, \rightsquigarrow, \mathcal{V}$, val,$\Delta\rangle$, where $\mathcal{A}$ is a finite set of arguments, $\rightsquigarrow$ is an attack relation over $\mathcal{A} \times \mathcal{A}, \mathcal{V}$ is a nonempty set of values, val $: \mathcal{A} \rightarrow 2^{\mathcal{V}}$ returns the set of values promoted by each argument, and $\Delta$ is the set of possible audiences. An audience specific argumentation framework is a five-tuple, $V A F_{\delta}=\left\langle\mathcal{A}, \rightsquigarrow, \mathcal{V}\right.$, val, $\left.\succ_{\delta}\right\rangle$, where $\delta \in \Delta$ is an audience and $\succ_{\delta}$ is a partial order over $\mathcal{V}$.

In this paper we consider audience specific argumentation framework and denote it $\left\langle\mathcal{A}, \rightsquigarrow, \mathcal{V}\right.$, val,$\left.\succ_{\mathcal{V}}\right\rangle$. We suppose that an argument promotes at least one value. Different ways have been proposed to compute a preference relation over $\mathcal{A}$ given $\succ_{\mathcal{V}}$. We refer the reader to [3, 14]. One may for instance use the following definition:

$$
\forall a, b \in \mathcal{A}, \quad a \succ b \quad \text { iff } \quad \exists v \in \operatorname{val}(a) \forall v^{\prime} \in \operatorname{val}(b) \quad v \succ_{\mathcal{V}} v^{\prime} .
$$

\subsection{Argumentation Framework with Varied-Strength Defeats}

Strength of defeat relations has been incorporated in argumentation framework in two ways: a qualitative relative way by means of a partial preorder [19, 20] and a quantitative way by means of a numerical function [9]. As far as the present paper is concerned, we follow the second modeling.

Definition 4. [9] An argumentation framework with varied-strength defeats (AFV) is a 3-tuple $\langle\mathcal{A}, \rightarrow$, VDef $\rangle$ where $\langle\mathcal{A}, \rightarrow\rangle$ is a Dung's argumentation framework and VDef is a function defined from $\rightarrow$ to $(0,1]$.

For simplicity, we consider the interval $(0,1]$ but any bipolar linearly ordered scale with top, bottom and neutral elements can be used as well. $V D e f(a, b)$ is the degree of the statement " $a$ defeats $b$ " being true. Values $0, \frac{1}{2}$ and 1 for $\operatorname{VDef}(a, b)$ mean that the validity of the previous statement is certainly false, unknown and certainly true respectively. We say that $a$ defeats $b$ w.r.t. $\langle\mathcal{A}, \rightarrow, V D e f\rangle$ iff $a \rightarrow b$.

Extensions are also defined from the conflict-freeness and defense. Conflict-freeness is defined as for $\langle\mathcal{A}, \rightarrow\rangle$. Defense is however extended to the valued case. When $b \rightarrow$ $a$ and $c \rightarrow b$, the strength of defeats should play a role in the definition of the defense since $c$ is considered as a "serious" defender of $a$ if the defeat of $c$ on $b$ is at least as strong as the defeat of $b$ on $a$. The set $A \subseteq \mathcal{A}$ defends $a \in \mathcal{A}$ w.r.t. $\langle\mathcal{A}, \rightarrow, V D e f\rangle$ iff for all $b \in \mathcal{A}$ such that $b \rightarrow a$, there exists $c \in A$ with [19]:

$$
c \rightarrow b \text { and } \operatorname{VDef}(c, b) \geq \operatorname{VDef}(b, a) .
$$

Let us now describe an instantiation of this framework where the valued defeat relation is derived from a valued preference relation $P: \mathcal{A} \times \mathcal{A} \rightarrow[0,1] . P(a, b)$ is the degree of the statement " $a$ is strictly preferred to $b$ " being true. 
Definition 5. [13] $A$ valued preference-based argumentation framework (VPAF) 1 is a 3-tuple $\langle\mathcal{A}, \rightsquigarrow, P\rangle$ where $\mathcal{A}$ is the set of arguments, $\rightsquigarrow \subseteq \mathcal{A} \times \mathcal{A}$ is a binary attack relation and $P$ is a function defined from $\mathcal{A} \times \mathcal{A}$ to $[0,1]$.

$\mathrm{A} \operatorname{VPAF}\langle\mathcal{A}, \rightsquigarrow, P\rangle$ represents an argumentation framework with varied-strength defeats $\langle\mathcal{A}, \rightarrow, V D$ ef $\rangle$ iff $a \rightarrow b$ if $a \rightsquigarrow b$ and $P(b, a)<1, \operatorname{VDef}(a, b)=1-P(b, a)$ if $a \rightarrow b$. Lastly, $\operatorname{VDef}(a, b)=0$ otherwise. An interesting case is when $P$ is derived from a valuation function $w$ over the arguments. A suitable expression of $P$ is $P(a, b)=w(a)-w(b)$ if $w(a)>w(b)$ and $P(a, b)=0$ else [13]. This gives

$$
\begin{aligned}
& a \rightarrow b \quad \text { if } \quad a \rightsquigarrow b \text { and }[w(a)>0 \text { or } w(b)<1], \\
& \operatorname{VDef}(a, b)=\min (1+w(a)-w(b), 1) \text { if } a \rightarrow b .
\end{aligned}
$$

\section{Arguing with Collective Defeat Relations}

\subsection{Collective Argumentation Framework}

Dung's framework has been extended with a defeat relation between sets of arguments.

Definition 6. [21] A collective argumentation framework is a pair $\langle\mathcal{A}, \rightrightarrows\rangle$ where $\mathcal{A}$ is a set of arguments and $\rightrightarrows \subseteq 2^{\mathcal{A}} \times 2^{\mathcal{A}}$ is the defeat relation, with, for $A \subseteq \mathcal{A}$ and $B \subseteq \mathcal{A}$, notation $A \rightrightarrows B$ means that the arguments in $A$ jointly defeat $B$.

The authors of [21] argue that there is no need to define defeat of a subset of arguments on another subset of arguments. In fact, they interpret $A \rightrightarrows B$ as $A \rightrightarrows\{b\}$ for every $b \in B$. Hence it is sufficient to see $\rightrightarrows$ as a subset of $2^{\mathcal{A}} \times \mathcal{A}$. This definition implicitly means that if $A \rightrightarrows\{b\}$ for every $b \in B$ then $A \rightrightarrows B$. However this interpretation may not be sufficient in many situations.

Example 2 (Example 1 cont.). $a$ defeats $b$ as the value promoted by $a$ is more important than that promoted by $b$. Likewise, $a$ defeats arguments $c$ and $d$. On the other hand, one may conceive that $a$ does not defeat the set of arguments $\{b, c, d\}$ since the values promoted by these arguments are collectively stronger than the value promoted by $a$.

The previous example indicates that defining defeats among subsets of arguments is important since the fact that $a$ defeats $b, c$ and $d$ considered separately does not necessarily imply that $a$ defeats $b, c$ and $d$ as a whole.

We don't define the meaning of $A$ "jointly" defeats $B$ at this stage. We borrowed collective argumentation framework from [21] as it nicely models our needs. However our interpretation of joint defeat differs from that proposed in [21], as we will see later. In [21] $A$ jointly defeats $B$ is interpreted as "arguments in $A$ do not separately defeat arguments in $B$ but considered together they do".

\footnotetext{
${ }^{1}$ Valued preference-based argumentation framework must not be confused with value-based argumentation framework [3]. In the latter, arguments promote values which may be point of views, decisions, opinions, etc. Then a preference relation over the set of arguments is derived from a preference relation over the values. In the former, the preference relation over the set of arguments is valued, i.e. it expresses preferences with varied strength, as we will see later.
} 
A set $A$ of arguments is conflict-free if there is no subsets $A^{\prime}, A^{\prime \prime} \subseteq A$ such that $A^{\prime} \rightrightarrows A^{\prime \prime}$. Let $A, B, C \subseteq \mathcal{A}$. We say that $C \subseteq \mathcal{A}$ defends $A \subseteq \mathcal{A}$ if $\forall B \subseteq \mathcal{A}$ with $B \rightrightarrows A$ we have $C \rightrightarrows B$. The semantics of acceptability can be defined from the concepts of conflict-freeness and defense as usual.

Relation $\rightrightarrows$ shall satisfy some monotonicity conditions: for all $A, B \subseteq \mathcal{A}$

$$
\forall B^{\prime} \subseteq B, \quad \text { if } A \rightrightarrows B \text { then } A \rightrightarrows B^{\prime} .
$$

Therefore we recover the interpretation of $A \rightrightarrows B$ given in [21]. However we do not necessarily have that $A \rightrightarrows B$ if $A \rightrightarrows B^{\prime}, \forall B^{\prime} \subseteq B, B^{\prime} \neq B$.

\subsection{Arguing with Collective Varied Defeats}

In this section we extend the collective argumentation framework defined in the previous subsection with a varied defeat relation.

Definition 7. A collective argumentation framework with varied defeats is a triplet $\langle\mathcal{A}, \rightrightarrows, \overline{V D e f}\rangle$ where $\mathcal{A}$ is a set of arguments and $\rightrightarrows \subseteq 2^{\mathcal{A}} \times 2^{\mathcal{A}}$ is a defeat relation and $\overline{V D e f}$ is a function from $\rightrightarrows$ to $(0,1]$.

$\overline{V D e f}(A, B)$ is the degree of credibility of statement " $A$ defeats $B$ ".

A set $A$ of arguments is conflict-free if there is no $A^{\prime}, A^{\prime \prime} \subseteq A$ such that $A^{\prime} \rightrightarrows A^{\prime \prime}$. We say that $C \subseteq \mathcal{A}$ defends $A \subseteq \mathcal{A}$ if for all $B \subseteq \mathcal{A}$ such that $B \rightrightarrows A$, there exists $C^{\prime} \subseteq C$ such that $C^{\prime} \rightrightarrows B$ and $\overline{V D e f}\left(C^{\prime}, B\right) \geq \overline{V D e f}(B, A)$. The semantics of acceptability can be defined from the concepts of conflict-freeness and defense as usual.

$\overline{V D e f}$ shall satisfy some monotonicity condition. For all $A, B, A^{\prime}, B^{\prime} \subseteq \mathcal{A}$

$$
\text { if } A^{\prime} \subseteq A, B^{\prime} \supseteq B, A \rightrightarrows B \text { and } A^{\prime} \rightrightarrows B^{\prime} \text { then } \overline{V D e f}\left(A^{\prime}, B^{\prime}\right) \leq \overline{V D e f}(A, B) \text {. }
$$

Indeed the more arguments we add to $A$ the stronger the defeat, and the more arguments we add to $B$ the weaker the defeat.

\section{Extended Value-Based Argumentation Framework}

In standard value-based argumentation framework arguments fully promote a subset of values in $\mathcal{V}$ [3]. In many applications however, arguments support values with various strengths. In Example 1, argument $d$ promotes only partly value pol. Hence function val is refined in the following way.

Definition 8. For $a \in \mathcal{A}$, we define $f: \mathcal{A} \times \mathcal{V} \rightarrow[0,1]$ such that $f(a, v)$ is the degree to which argument $a \in \mathcal{A}$ supports value $v \in \mathcal{V}$.

The aim of this section is to extend single defeats (i.e., an argument defeats an argument) to collective defeats (i.e., a set of arguments defeats a set of arguments). These defeats will hold with degrees that will be derived from $f$. 


\subsection{Construction}

The main question we face to define $\overline{V D e f}$ is to what extent different arguments can produce a stronger defeat than each argument can do individually. In some sense, they have some complementarity among themselves. The key idea is that each argument may support a different value and that the audience is much more convinced by a set of relevant values than by only one of them.

Example 3 (Example 1 cont.). The audience may say that value health is more important than any other value eco, env or pol. Hence $a$ defeats $b, c, d$ and none of $b, c, d$ defeats $a$. On the other hand, arguments $b, c, d$ promote three different values and, one may conceive that $b, c, d$ together defeat $a$.

In order to define $\overline{V D e f}(A, B)$, we need to extend the boolean preference relation $\succ_{\mathcal{V}}$ over $\mathcal{V}$ to a valued preference relation over $2^{\mathcal{V}}$. We represent this preference relation by a numerical function $\mu: 2^{\mathcal{V}} \rightarrow \mathbb{R}^{+}$. For $V \subseteq \mathcal{V}, \mu(V)$ is the strength of the preference if all values in $V$ are completely promoted and the remaining values are not promoted at all. This set function, called a capacity, shall satisfy some properties [6].

Definition 9. A capacity on $\mathcal{V}$ is a set function $\mu: 2^{\mathcal{V}} \rightarrow \mathbb{R}_{+}$satisfying two properties:

- (monotonicity) $\mu(V) \leq \mu\left(V^{\prime}\right)$ for all $V, V^{\prime} \subseteq \mathcal{V}$ with $V \subseteq V^{\prime}$,

- (boundary condition) $\mu(\emptyset)=0$ and $\mu(\mathcal{V})=1$.

The monotonicity condition will serve in the definition of a strength of defeats: the more values a set of arguments supports, the stronger the defeat. The boundary condition essentially says that the audience is not convinced by a set of arguments if they do not support any value 2 . Hence the values represent all possible stakes and points of view the audience may believe in. Normalization condition $\mu(\mathcal{V})=1$ comes from the fact that the strength of defeat is bounded by 1 .

Note that capacity $\mu$ is a refinement of order $\succ_{\mathcal{V}}$ :

$$
\forall v, v^{\prime} \in \mathcal{V} \text {, if } v \succ_{\mathcal{V}} v^{\prime} \quad \text { then } \quad \forall V \subseteq \mathcal{V} \backslash\left\{v, v^{\prime}\right\} \quad \mu(V \cup\{v\})>\mu\left(V \cup\left\{v^{\prime}\right\}\right) .
$$

This property is similar to responsiveness defined by Roth [23] (see also [4]).

On the basis of the above definitions, we define an extended value-based argumentation framework in the following way:

Definition 10. An extended value-based argumentation framework is a five-tuple, $\langle\mathcal{A}, \rightsquigarrow, \mathcal{V}, f, \mu\rangle$, where $\mathcal{A}$ is a finite set of arguments, $\rightsquigarrow \mathcal{A} \times \mathcal{A}$ is an attack relation, $\mathcal{V}$ is a nonempty set of values, $f$ is a function from $\mathcal{A} \times \mathcal{V}$ to $[0,1]$, and $\mu$ is a capacity over $\mathcal{V}$.

We are going to derive a collective argumentation framework with varied defeats $\langle\mathcal{A}, \rightrightarrows$ $, \overline{V D e f}\rangle$ (see Definition 7). We extend $w$ and relations (3) and (4). To this end, we define a valuation $G: 2^{\mathcal{A}} \rightarrow[0,1]$ of subsets of arguments. More precisely, it evaluates the

\footnotetext{
${ }^{2}$ Arguments in $\mathcal{A}$ are supposed to promote at least one value, but one may imagine other arguments promoting no value in $\mathcal{V}$.
} 
strength of a set of arguments. This strength will be used to evaluate the degree of defeat between two subsets of arguments. $G(A)$ (with $A \subseteq \mathcal{A}$ ) depends on the values promoted by arguments in $A$ (see function $f$ ) and the strength of these values (see function $\mu$ ). First of all, extending (3), the defeat relation $\rightrightarrows$ is defined as follows:

$$
\begin{aligned}
A \rightrightarrows B \text { if } & (G(A)>0 \text { or } G(B)<1) \text { and } \\
& {[\forall b \in B \exists a \in A \quad a \rightsquigarrow b] \text { and }[\forall a \in A \exists b \in B \quad a \rightsquigarrow b] }
\end{aligned}
$$

where Following (4), the intensity of the defeat is given by

$$
\overline{\operatorname{VDef}}(A, B)=\min (1+G(A)-G(B), 1) \quad \text { if } A \rightrightarrows B
$$

According to these definitions, if $A \rightrightarrows B$ then $\overline{\operatorname{VDef}}(A, B)>0$, as required by Definition 7 The next two subsections are devoted to the definition of the function $G$.

\subsection{Computing $G(A)$ : Case When $A$ Is a Singleton}

Consider in this section the case where $A=\{a\} . G(\{a\})$ depends only on the degree to which values are supported by $a$ (i.e. on $\{f(a, v) \mid v \in \mathcal{V}\}$ ) as well as on the strength $\mu$ of values. Hence there exists a function denoted by $F_{\mu}: \mathbb{R}_{+}^{\mathcal{V}} \rightarrow \mathbb{R}_{+}$(to be determined) such that:

$$
G(\{a\})=F_{\mu}(\{f(a, v) \mid v \in \mathcal{V}\}) .
$$

We will use an axiomatic approach to get $F_{\mu}$ from a set of wished properties on $F_{\mu}$.

- Properties of the Function $\boldsymbol{F}_{\boldsymbol{\mu}}$ We already justified monotonicity condition on the capacity (see Definition 9). This condition can be extended to $F_{\mu}$. If the degree to which an argument supports a value increases, $F_{\mu}$ shall not decrease.

Increasingness (In): $\forall x, y \in \mathbb{R}^{\mathcal{V}}$, if $x_{v} \leq y_{v} \forall v \in \mathcal{V}$ then $F_{\mu}(x) \leq F_{\mu}(y)$.

Element $x_{v}$ (resp. $y_{v}$ ) represents the degree to which an argument $a$ (resp. another argument $b$ ) promote values $v$ in $\mathcal{V}$. As argument $b$ promotes every value at least as well as $a\left(x_{v} \leq y_{v}\right.$ for every $\left.v \in \mathcal{V}\right)$, the valuation of $b$ should not be lower. $F_{\mu}(x) \leq F_{\mu}(y)$ derives from $G(\{a\}) \leq G(\{b\})$.

In the previous subsection, we have interpreted $\mu(V)$ as the strength of preference if all values in $V$ are completely promoted and the remaining ones are not. Formally, we write:

$$
\text { Properly Weighted (PW): } F_{\mu}(\underbrace{1, \cdots, 1}_{v \in V}, \underbrace{0, \cdots, 0}_{v \notin V})=\mu(V), \forall V \subseteq \mathcal{V} \text {. }
$$

From (PW), if $\mu$ is multiplied by a number then the resulting strength is also multiplied by the same factor: $F_{\gamma \mu}(x)=\gamma F_{\mu}(x)$ for any $\gamma \in \mathbb{R}$. As a capacity $\mu$ may be provided by an expert, if another expert provides $\mu^{\prime}$ then one may combine $\mu$ and $\mu^{\prime}$ with a linear transformation $\gamma \mu+\delta \mu^{\prime}(\gamma, \delta \in \mathbb{R})$. Then it is reasonable that the overall 
aggregation function equals the same linear transformation of the aggregation for the two decision makers:

Linearity w.r.t. the Measure (LM): For all $x \in \mathbb{R}^{\mathcal{V}}$ and $\gamma, \delta \in \mathbb{R}$,

$$
F_{\gamma \mu+\delta \mu^{\prime}}(x)=\gamma F_{\mu}(x)+\delta F_{\mu^{\prime}}(x) .
$$

The numerical values of $f(a,$.$) correspond to an interval scale in the sense of measure-$ ment theory [16]. An interval scale is given up to an affine transformation. Hence $F_{\mu}$ shall be invariant under any affine transformation. However, as all degrees $f(a, v)$ for all $v$ correspond to the same scale, the same transformation shall be applied to all values in $\mathcal{V}$. Starting from (PW), we impose this invariance property only on situation where each value is either completely supported or not supported at all.

Stability for the admissible Positive Linear transformations (weak SPL):

For all $V \subset \mathcal{V}, \alpha>0$, and $\beta \in \mathbb{R}$,

$$
F_{\mu}(\underbrace{(\alpha+\beta), \cdots,(\alpha+\beta)}_{v \in V}, \underbrace{\beta, \cdots, \beta}_{v \notin V})=\alpha F_{\mu}(\underbrace{1, \cdots, 1}_{v \in V}, \underbrace{0, \cdots, 0}_{v \notin V})+\beta .
$$

This axiom is a weak version of the axiom (SPL) introduced by Marichal [18] : For all $x \in \mathbb{R}^{\mathcal{V}}, \alpha>0$, and $\beta \in \mathbb{R}, F_{\mu}(\alpha x+\beta)=\alpha F_{\mu}(x)+\beta$.

Example 4 (Example 1 cont.). The following values of $\mu$ are supposed given:

$$
\begin{array}{lll}
\mu(\emptyset)=0 & \mu(\{\text { health }\})=0.6 & \mu(\{\text { eco }\})=0.2 \\
\mu(\{\text { env }\})=0.1 & \mu(\{\text { pol }\})=0.05 & \mu(\{\text { eco,env }\})=0.5 \\
\mu(\{\text { eco }, \text { pol }\})=0.3 & \mu(\{\text { env }, \text { pol }\})=0.2 & \mu(\{\text { eco, env }, \text { pol }\})=0.9 \\
\mu(\{\text { health }, \text { eco }, \text { env }, \text { pol }\})=1 & &
\end{array}
$$

We note that there is a strong positive synergy among values eco, env, pol as

$$
\mu(\{e c o, e n v, p o l\})>\mu(\{e c o\})+\mu(\{e n v\})+\mu(\{p o l\}) .
$$

These three values together are more important than value health alone.

- Function $\boldsymbol{F}_{\boldsymbol{\mu}}$ vs Choquet Integral. Now that we have given properties of $F_{\mu}$, we show that this function is already at work in multiple criteria decision and known as Choquet integral [6]. The Choquet integral is a generalization of the commonly used weighted sum.

Definition 11. Let $\mu$ be a capacity on $\mathcal{V}$, with $|\mathcal{V}|=n$. Let $x=\left(x_{1}, \cdots, x_{n}\right) \in \mathbb{R}^{\mathcal{V}}$. The discrete Choquet integral of $x$ with respect to $\mu$ is defined by

$$
C_{\mu}(x)=\sum_{i=1}^{n}\left(x_{(i)}-x_{(i-1)}\right) \mu(\{(i), \cdots,(n)\}),
$$

with $x_{(0)}=0$, and where $(1), \ldots,(n)$ indicate that the indices have been permuted so that $0 \leq x_{(1)} \leq \cdots \leq x_{(n)}$.

Example 5. Let us illustrate Def. 11 on $x=(0,0.6,1,0.1)$. The worse score of $x$ is its first component (i.e. $(1)=1$ ), the second worse score of $x$ is its last component (i.e. $(2)=4)$, the third worse score of $x$ is its second component (i.e. $(3)=2)$ and the best score of $x$ is its third component (i.e. $(4)=3)$. Hence $C_{\mu}(x)=\left(x_{1}-0\right) \mu(\{1,2,3,4\})+$ $\left(x_{4}-x_{1}\right) \mu(\{2,3,4\})+\left(x_{2}-x_{4}\right) \mu(\{2,3\})+\left(x_{3}-x_{2}\right) \mu(\{3\})$. 
Besides, the Choquet integral can model typical human behavior such as the veto. This operator is also able to model the importance of values and the interaction between values. Conversely, the Choquet integral can be interpreted in terms of the importance of values, the interaction between values, and veto [10, 11].

Proposition 1 ([17]). $F_{\mu}$ satisfies (LM), (In), (PW) and (weak SPL) if and only if $F_{\mu} \equiv C_{\mu}$ in $\mathbb{R}^{\mathcal{V}}$.

The proof of this proposition can be found in [17]. Proposition 1 shows that if one agrees on properties (LM), (In), (PW) and (weak SPL), then he shall use the Choquet integral w.r.t. $\mu$, namely $G(\{a\})=C_{\mu}(\{f(a, v) \mid v \in \mathcal{V}\})$ (see (9)).

\subsection{Computing $G(A)$ : Case Where $A$ Is Composed of Several Arguments}

When $A$ is not reduced to a singleton, we generalize the construction given in Subsection 4.2. Function $F_{\mu}$ can still be used to compute $G(A)$. We denote by $x_{f, A}(v)$ the degree to which all arguments in $A$ promote together value $v$, with $x_{f, A} \in[0,1]^{\mathcal{V}}$. Hence (9) is generalized as follows:

$$
G(A)=F_{\mu}\left(x_{f, A}\right) .
$$

For $v \in \mathcal{V}, x_{f, A}$ is derived from $\{f(a, v) \mid a \in A\}$. A maximum function could work: $x_{f, A}(v)=\max _{a \in A} f(a, v)$. However, for the same maximal number of the individual $f(a, v)$, this maximal number could be reinforced if $f\left(a, v^{\prime}\right)$ is also large for another value $v^{\prime}$. A t-conorm 3 denoted by $\oplus$ could be used to express this reinforcement property. Note that $\alpha \oplus 0=\alpha$ for all $\alpha \in[0,1]$. Hence

$$
\forall v \in \mathcal{V} \quad x_{f, A}(v)=\oplus\{f(a, v) \mid a \in A\}
$$

Example 6 (Example 3 continued). Assume the values of $f$ are: $f(a$, health $)=1$, $f(b, e c o)=1, f(c, e n v)=1$ and $f(d, p o l)=.5$. All other values of $f(.,$.$) are equal to$ 0 . Let us compute the value of $G$ for several subsets of arguments

$$
\begin{aligned}
& G(\{a\})=C_{\mu}(1,0,0,0)=\mu(\text { health })=0.6 \\
& G(\{b\})=C_{\mu}(0,1,0,0)=\mu(\text { eco })=0.2 \\
& G(\{b, c\})=C_{\mu}(0,1,1,0)=\mu(\{\text { eco, env }\})=0.5 \\
& G(\{b, c, d\})=C_{\mu}(0,1,1,0.5)=0.5 \mu(\{\text { eco, env }\})+0.5 \mu(\{\text { eco, env }, \text { pol }\})=0.7 .
\end{aligned}
$$

Let us now consider the following subsets of arguments

- Set $\{b, c\}$ is conflict-free but does not defend itself. Indeed for the attack by $a$, we have $\{a\} \rightrightarrows\{b, c\},\{b, c\} \rightrightarrows\{a\}$ but (see $[8$ )

$$
\overline{V D e f}(\{b, c\},\{a\})=0.9<\overline{V D e f}(\{a\},\{b, c\})=1
$$

\footnotetext{
${ }^{3}$ A function $\oplus:[0,1] \times[0,1] \rightarrow[0,1]$ is called a t-conorm (triangular conorm) if it satisfies $\oplus(0, x)=x$ for all $x \in[0,1]$ (neutral element), $\oplus(x, y)=\oplus(y, x)$ for all $x, y \in[0,1]$ (commutativity), $\oplus(x, y) \leq \oplus(u, v)$ for all $0 \leq x \leq u \leq 1$ and $0 \leq y \leq v \leq 1$ (monotonicity), and $\oplus(x, \oplus(y, z))=\oplus(\oplus(x, y), z)$ for all $x, y, z \in[0,1]$ (associativity) [15].
} 
- Set $\{a\}$ is conflict-free but does not defend itself. Indeed for the attack by $b, c, d$, we have $\{b, c, d\} \rightrightarrows\{a\},\{a\} \rightrightarrows\{b, c, d\}$ but (see (8))

$$
\overline{V D e f}(\{a\},\{b, c, d\})=0.9<\overline{\operatorname{VDef}}(\{b, c, d\},\{a\})=1
$$

- Set $\{b, c, d\}$ is conflict-free and defends itself. Indeed for the attack by $a$, we have $\{a\} \rightrightarrows\{b, c, d\},\{b, c, d\} \rightrightarrows\{a\}$ and (see (8))

$$
\overline{\operatorname{VDef}}(\{b, c, d\},\{a\})=1>\overline{\operatorname{VDef}}(\{a\},\{b, c, d\})=0.9 .
$$

Hence $\{b, c, d\}$ is the unique set of admissible arguments.

Assume now that argument $d$ is removed from $\mathcal{A}$, so $\mathcal{A}=\{a, b, c\}$. In this case, $\{a\}$ is an extension as it defends itself from the attack of $\{b, c\}$ (since $\{b, c\} \rightrightarrows\{a\},\{a\} \rightrightarrows$ $\{b, c\}$ and $\overline{V D e f}(\{a\},\{b, c\})=1>\overline{\operatorname{VDef}}(\{b, c\},\{a\})=0.9)$. Thus $a$ becomes acceptable as $b, c$ are not sufficiently strong compared to $a$.

\subsection{Particular Case: No Interaction among Values}

Let us see how relation (2) can be satisfied in our framework. Condition (2) considers the case where $f$ takes only values 0 or 1 . Hence one can define $\operatorname{val}$ from $f$ by: $\operatorname{val}(a)=$ $\{v \in \mathcal{V}, f(a, v)=1\}$ for every $a \in \mathcal{A}$. Then by (PW), we have for every $a \in \mathcal{A}$, $G(\{a\})=\mu(\operatorname{val}(a))$. Intuitively one feels that relation (2) can be translated in terms of capacity $\mu$ in the following way: for all $V, V^{\prime} \subseteq \mathcal{V}$

$$
\mu(V)>\mu\left(V^{\prime}\right) \quad \text { iff } \quad \exists v \in V \forall v^{\prime} \in V^{\prime} \quad \mu(\{v\})>\mu\left(\left\{v^{\prime}\right\}\right) .
$$

There is no possible cumulative effect (synergy) among the values in this case. This condition is satisfied for instance when $\mu(V)=\max _{v \in V} \mu(\{v\})$, which corresponds to a possibility measure. The next result shows that under (13), collective defeats will not bring added-value to single defeats.

Proposition 2. Assume that values are either completely promoted or not all by arguments. Assume furthermore that relation (13) holds. Under (8), we have

- $\forall A, B \subseteq \mathcal{A}$, if $A \rightrightarrows B$ (i.e. $\overline{V D e f}(A, B)>0$ ), then there exists a $\in A$ such that $\{a\} \rightrightarrows B$ (i.e. $\overline{V D e f}(\{a\}, B)>0)$;

- $\forall A \subseteq \mathcal{A}, b_{1}, \ldots, b_{p} \in \mathcal{A}$, if $A \rightrightarrows b_{1}$ (i.e. $\left.\overline{V D e f}\left(A,\left\{b_{1}\right\}\right)>0\right), \ldots, A \rightrightarrows b_{p}$ (i.e. $\left.\overline{V D e f}\left(A,\left\{b_{p}\right\}\right)>0\right)$, then $A \rightrightarrows\left\{b_{1}, \ldots, b_{p}\right\}$ (i.e. $\left.\overline{\operatorname{VDef}}\left(A,\left\{b_{1}, \ldots, b_{p}\right\}\right)>0\right)$.

The proof of this proposition is omitted due to the lack of space. This proposition shows that our framework is general and can encompass the standard case of relation (2). More precisely, condition (2) is translated into (13). Proposition 2] shows that under (13), there exists an argument in the attacking set that defeat the attacked set, and if the attacking set defeats each argument in a set, it defeats the set collectively. This corresponds well to the idea behind (2). 


\section{Conclusion}

In some argument-based applications, arguments need to collectively interact. More precisely, defeat relation is defined among sets of arguments. Moreover this relation has varied strengths due to the fact that arguments promote some values (decision, point of view, etc) with varied strengths. In this paper we developed an argumentation framework extending value-based argumentation framework [3] in order to cope with the above considerations. The strength of defeat $\overline{V D e f}(A, B)$ of a subset $A$ of arguments over another subset $B$ depends of the values promoted by $A$ and $B$. The synergy among the values is encoded in a capacity $\mu$ defined on the set of values. As arguments may promote only partly the values, the strength of all arguments in $A$ collectively considered is obtained by using an aggregation function depending on capacity $\mu$. We define desired properties for the aggregation function. We show that this function obeying the corresponding properties is Choquet integral.

Our framework may be applicable to model coalition-based problems where sets of arguments correspond to coalitions [5]. For future work we intend to compare our approach with the accrual of arguments proposed in [22]. We also intend to consider other definitions of defense as suggested in [7]. Lastly, qualitative aggregation functions such as the Sugeno integral will be also considered.

\section{References}

1. Amgoud, L., Cayrol, C.: Inferring from inconsistency in preference-based argumentation frameworks. International Journal of Approximate Reasoning 29(2), 125-169 (2002)

2. Amgoud, L., Cayrol, C., LeBerre, D.: Comparing arguments using preference orderings for argument-based reasoning. In: ICTAI 1996, pp. 400-403 (1996)

3. Bench-Capon, T.J.M.: Persuasion in practical argument using value-based argumentation frameworks. Journal of Logic and Computation 13(3), 429-448 (2003)

4. Bossert, W.: Preference extension rules for ranking sets of alternatives with a fixed cardinality. Theory and Decision 39, 301-317 (1995)

5. Bulling, N., Dix, J., Chesñevar, C.: Modelling coalitions: Atl + argumentation. In: AAMAS, pp. 681-688 (2008)

6. Choquet, G.: Theory of capacities. Annales de l'Institut Fourier 5, 131-295 (1953)

7. Coste-Marquis, S., Konieczny, S., Marquis, P., Ouali, M.: Weighted attacks in argumentation frameworks. In: KR (2012)

8. Dung, P.M.: On the acceptability of arguments and its fundamental role in non-monotonic reasoning, logic programming and n-person games. Artificial Intelligence 77, 321-357 (1995)

9. Dunne, P.E., Hunter, A., McBurney, P., Parsons, S., Wooldridge, M.: Inconsistency tolerance in weighted argument systems. In: AAMAS, pp. 851-858 (2009)

10. Grabisch, M.: The application of fuzzy integrals in multicriteria decision making. European Journal of Operational Research 89, 445-456 (1996)

11. Grabisch, M., Labreuche, C.: A decade of application of the Choquet and Sugeno integrals in multi-criteria decision aid. Annals of Operation Research 175, 247-286 (2010)

12. Kaci, S.: Refined preference-based argumentation frameworks. In: COMMA, pp. 299-310 (2010)

13. Kaci, S., Labreuche, C.: Arguing with valued preference relations. In: Liu, W. (ed.) ECSQARU 2011. LNCS, vol. 6717, pp. 62-73. Springer, Heidelberg (2011) 
14. Kaci, S., van der Torre, L.: Preference-based argumentation: Arguments supporting multiple values. International Journal of Approximate Reasoning 48, 730-751 (2008)

15. Klement, E., Mesiar, R., Pap, E.: Triangular Norms. Kluwer, Dordrecht (2000)

16. Krantz, D.H., Luce, R.D., Suppes, P., Tversky, A.: Foundations of measurement. Additive and Polynomial Representations, vol. 1. Academic Press (1971)

17. Labreuche, C., Grabisch, M.: The Choquet integral for the aggregation of interval scales in multicriteria decision making. Fuzzy Sets \& Systems 137, 11-26 (2003)

18. Marichal, J.-L.: An axiomatic approach of the discrete Choquet integral as a tool to aggregate interacting criteria. IEEE Tr. on Fuzzy Systems 8(6), 800-807 (2000)

19. Martínez, D.C., García, A.J., Simari, G.R.: An abstract argumentation framework with varied-strength attacks. In: KR, pp. 135-144 (2008)

20. Martínez, D.C., García, A.J., Simari, G.R.: Strong and weak forms of abstract argument defense. In: COMMA, pp. 216-227 (2008)

21. Nielsen, S.H., Parsons, S.: A generalization of dung's abstract framework for argumentation: Arguing with sets of attacking arguments. In: Maudet, N., Parsons, S., Rahwan, I. (eds.) ArgMAS 2006. LNCS (LNAI), vol. 4766, pp. 54-73. Springer, Heidelberg (2007)

22. Prakken, H.: A study of accrual of arguments, with applications to evidential reasoning. In: ICAIL, pp. 85-94 (2005)

23. Roth, A.: The college admissions problem is not equivalent to the marriage problem. Journal of Economic Theory 36, 277-288 (1985)

24. Simari, G.R., Loui, R.P.: A mathematical treatment of defeasible reasoning and its implementation. Artificial Intelligence 53, 125-157 (1992) 